\title{
Astragalus saponins inhibit cell growth, aerobic glycolysis and attenuate the inflammatory response in a DSS-induced colitis model
}

\author{
HAILONG GUO $^{1 *}$, BOSHUN WAN $^{2 *}$, JIANSONG WANG $^{3 *}$, JIE ZHANG $^{1}$, WENFEI YAO ${ }^{4}$ and ZHIYONG SHEN ${ }^{1}$ \\ ${ }^{1}$ The Southern Division of Renji Hospital, Shanghai Jiao Tong University School of Medicine, Shanghai 201112; \\ ${ }^{2}$ Department of General Surgery, Jiading District Central Hospital Affiliated Shanghai University of Medicine and \\ Health Sciences, Shanghai 201800; ${ }^{3}$ Department of Thoracic Surgery, Weifang Medical University, Weifang 261000, \\ Shandong; ${ }^{4}$ The Northern Division of Ruijin Hospital, Shanghai Jiaotong University School of Medicine, \\ Shanghai 200135, P.R. China
}

Received June 10, 2018; Accepted November 26, 2018

DOI: 10.3892/ijmm.2018.4036

\begin{abstract}
Recent studies have reported that Astragalus saponins (AST), extracted from the medicinal plant Astragalus membranaceus, possess anti-tumor and apoptosis-inducing abilities on various types of human cancer in vitro and in vivo. However, limited studies have explored how AST impacts glucose metabolism and growth conditions in vitro. The present study aimed to explore cell growth, proliferation, apoptosis and a series of glycolysis metabolic alterations associated with AST treatment in colorectal cancer (CRC) cells. MTT, a colony formation assay and flow cytometry demonstrated that AST dose-dependently inhibited cell viability and induced apoptosis. Glucose uptake and lactate production measurements revealed that AST could inhibit glycolysis metabolism and lactate production. Reverse transcription-quantitative polymerase chain reaction and western blot analysis identified that the expression levels of glycolytic enzymes were decreased by AST treatment in CRC cells. To uncover the possible impact of AST on inflammation and glucose metabolism in vivo, a dextran sulfate sodium (DSS)-induced colitis mouse model was established. Notably, AST could inhibit growth and glycolysis metabolism in CRC cells in vitro, and attenuate the inflammatory response and tumor-like aerobic glycolysis in the DSS-induced mouse model. The findings indicated that AST may have the capacity to resist tumor-associated
\end{abstract}

Correspondence to: Dr Zhiyong Shen, The Southern Division of Renji Hospital, Shanghai Jiao Tong University School of Medicine, 2000 Jiangyue Road, Minhang, Shanghai 201112, P.R. China E-mail: bioZyS@hotmail.com

${ }^{*}$ Contributed equally

Key words: astragalus saponins, colorectal cancer, aerobic glycolysis, inflammation, apoptosis, growth inflammation and maintain normal glucose homeostasis, suggesting that AST could be a novel therapeutic strategy in $\mathrm{CRC}$ treatment.

\section{Introduction}

Recent epidemiological studies have suggested that colorectal cancer (CRC) is one of the most common malignancies, and millions of new CRC cases are diagnosed worldwide every year (1). Incidence and mortality rates have been decreasing for several years because of historical changes in risk factors, including reduced smoking and red meat consumption and increased use of aspirin (2). Furthermore, knowledge regarding the initiation, promotion and progression of CRC has increased over recent years (2). However, no effective therapeutic strategy has been identified to provide a long-term cure. Notably, several factors have been identified to increase the risk of CRC, including duration and extent of colitis, family history of CRC and severity of histologic inflammation (3). Chronic inflammation has been increasingly demonstrated to contribute to all steps of tumor development (4).

Astragalus saponins (AST), extracted from the medicinal plant Astragalus membranaceus, are the main active constituent in Radix Astragali, and its anti-tumor effects have been investigated in various studies $(5,6)$. In China, AST has been commonly used as an immunomodulating agent in mixed herbal decoctions to treat the common cold, diarrhea, fatigue and anorexia, and has also been prescribed to patients with cardiac diseases (7). Several studies have reported that AST possesses anti-tumor and apoptosis-inducing effects on various types of human cancer in vitro and in vivo $(6,8-10)$. Furthermore, clinical studies have reported that AST polysaccharides could increase the effectiveness of platinum-based chemotherapy when combined with chemotherapy and improve the quality of life in patients with advanced non-small cell lung cancer $(7,11,12)$. However, few studies have demonstrated the impact of AST treatment on glucose metabolism and growth conditions in CRC cells. 
Considering this, the present study investigated the effect of AST treatment on cell viability and apoptosis in vitro. Additionally, the present study explored the effect of AST treatment on glycolysis metabolism, including glucose uptake, lactate production and expression of glycolytic enzymes. The findings of the present study may provide a new therapeutic strategy in CRC treatment.

\section{Materials and methods}

Cell culture. Human CRC cell lines HT-29 and SW620 were obtained from American Type Culture Collection (Manassas, VA, USA). Cells were respectively maintained in McCoy's 5A medium or L-15 medium (Thermo Fisher Scientific, Inc., Waltham, MA, USA) supplemented with $10 \%$ fetal bovine serum at $37^{\circ} \mathrm{C}$ in humidified tissue culture incubator containing $5 \% \mathrm{CO}_{2}$.

Preparation of AST. Radix Astragali [Astragalus membranaceus (Fisch.) Bunge var. mongolicus] was obtained from Shaanxi University of Chinese Medicine (Shanxi, China). The authenticity and quality of the crude herb were confirmed in the Quality Assurance Laboratory of the School of Chinese Medicine, Hong Kong Baptist University (Hong Kong, China) by microscopic and chromatographic analyses, as well as DNA finger-printing. To ensure consistency between batches, voucher specimens were kept at the herbarium center (Shanghai, China) for future reference. AST was extracted as described previously (13). In brief, $500 \mathrm{~g}$ of crude herb was refluxed in methanol for $1 \mathrm{~h}$ at room temperature. Following this, n-butanol was added to the reconstituted residue for phase separation to obtain the total AST. Butanol was removed in the rotary evaporator. The resulting residue was reconstituted with distilled water and lyophilized into dry powder. The dried and lyophilized powder was reconstituted in ultrapure water to form a $10 \mathrm{mg} / \mathrm{ml}$ stock and stored at $-20^{\circ} \mathrm{C}$.

MTT assay. HT-29 and SW620 cells were seeded in 96-well plates at a density of $1 \times 10^{4}$ cells/well and treated with AST at the indicated concentrations $(0,0.02,0.04,0.08,0.16$ and $0.20 \mathrm{mg} / \mathrm{ml})$ and time intervals $(0,12,24,48$ and $72 \mathrm{~h})$. Control cells received McCoy's 5A or L-15 treatment only. A sterile MTT solution (Sigma-Aldrich; Merck KGaA, Darmstadt, Germany) was added to each well, and the cells were incubated for an additional $4 \mathrm{~h}$ at $37^{\circ} \mathrm{C}$. The medium was removed and $150 \mu$ l dimethyl sulfoxide was added to dissolve the formazan crystals formed in the viable cells. Plates were examined at $570 \mathrm{~nm}$ using a microplate reader (Bio-Rad Laboratories, Inc., Hercules, CA, USA). At least three independent experiments were performed.

Colony formation assay. HT-29 and SW620 cells were seeded in 6-well plates at a density of 500 cells/well and treated with $50 \mu \mathrm{g} / \mathrm{ml}$ AST. Cells treated with $25 \mu \mathrm{g} / \mathrm{ml}$ 5-fluorouracil (5-Fu, Sigma-Aldrich; Merck KGaA) were used as a positive control group. The medium (McCoy's 5A or L-15) was refreshed every 2 days. Following two weeks of incubation at $37^{\circ} \mathrm{C}$, colonies were fixed with methanol in $15 \mathrm{~min}$ at room temperature and then stained with Giemsa solution (Sigma-Aldrich; Merck $\mathrm{KGaA}$ ) for 10-15 min at room temperature. Plates were washed with PBS, and colony formation was photographed and analyzed using ImageJ 1.48 (National Institutes of Health, Bethesda, MD, USA). Cloning efficiency is the number of colonies divided by the number of cells plated.

Apoptosis assay. To investigate the effect of AST treatment on cell apoptosis, cells treated with $50 \mu \mathrm{g} / \mathrm{ml}$ AST served as the experimental group. Untreated cells were used as a control group. Cell apoptosis was analyzed using an Annexin V/propidium iodide apoptosis detection kit (BD Biosciences, San Jose, CA, USA) according to the instructions of the manufacturer. Following $48 \mathrm{~h}$ of AST treatment, the cells were harvested, washed with cold PBS (pH 7.4), centrifuged at $400 \mathrm{x} \mathrm{g}$ at $4^{\circ} \mathrm{C}$ for $5 \mathrm{~min}$, and double-stained with Annexin V-Fluorescein isothiocyanate and propidium iodide in binding buffer [10 mM HEPES (pH 7.4), $140 \mathrm{mM} \mathrm{NaCl}$ and $2.5 \mathrm{mM} \mathrm{CaCl}_{2}$ ] for $15 \mathrm{~min}$ in the dark. The samples were analyzed with Attune NxT Software version 2.5 and a flow cytometer (Invitrogen Attune NxT; Thermo Fisher Scientific, Inc.).

Reverse transcription-quantitative polymerase change reaction ( $R T-q P C R)$. Total RNA was isolated using TRIzol reagent (Invitrogen; Thermo Fisher Scientific, Inc.) according to the manufacturer's instructions. Subsequenetly, cDNA was synthesized with an iScript Advanced cDNA Synthesis kit (Bio-Rad Laboratories, Inc.). PCR was performed in duplicate with a CFX96 PCR system and iTaq Universal SYBR-Green Supermix according to the manufacturer's instructions (Bio-Rad Laboratories, Inc.). PCR amplification conditions were as follows: 1 cycle of $95^{\circ} \mathrm{C}$ for $3 \mathrm{~min}$, followed by 40 cycles of $95^{\circ} \mathrm{C}$ for $10 \mathrm{sec}$ and $60^{\circ} \mathrm{C}$ for $22 \mathrm{sec}$. Primers used were as follows: Human (h) $\beta$-actin forward, $5^{\prime}$-CCT GGGCATGGAGTCCTGTG-3' and reverse, 5'-TCTTCATTG TGCTGGGTGCC-3'; (h)c-Myc forward, 5'-GGAGGAACA AGAAGATGAGGAAG-3' and reverse, 5'-AGGACCAGT GGGCTGTGAGG-3'; (h) lactate dehydrogenase A (LDH-A), forward, 5'-CCCCAGAATAAGATTACAGTTATTG-3' and reverse, 5'-GAGCAAGTTCATCTGCCAAGT-3'; (h) hexokinase 2 (HK2) forward, 5'-GATTGTCCGTAACATTCTCAT CGA-3' and reverse, 5'-TGTCTTGAGCCGCTCTGAGAT-3'; (h) Glut-1 forward, 5'-TCTGGGCATGTGCTTCCAGTA-3' and reverse, 5'-ATCGAAGGTCCGGCCTTTAGTC-3'; mouse (m) $\beta$-actin forward, 5'-ATGCCATCCTGCGTCTGG ACCTGGC-3' and reverse, 5'-AGCATTTGCGGTGCACGA TGGAGGG-3'; (m)c-Myc forward, 5'-TCTCCATCCTATGTT GCGGTC-3' and reverse, 5'-TCCAAGTAACTCGGTCAT CATCT-3'; (m)LDH-A forward, 5'-GCTCCCCAGAACAAG ATTACAG-3' and reverse, 5'-TCGCCCTTGAGTTTGTC TTC-3'; and (m)phosphof ructokinase (PFK)U forward, 5'-CCG AGGAGCGTACAAAGT-3' and reverse, 5'-CTGAGCGGT GGTGGTGAT-3'.

Western blot. HT-29 and SW620 cells were lysed in radioimmunoprecipitation assay lysis buffer (Beyotime Institute of Biotechnology, Shanghai, China). Following this, total protein concentration was determined using the BCA assay Kit (Pierce; Thermo Fisher Scientific, Inc.). Equal amounts of protein $(20 \mu \mathrm{g}$ per lane) were separated using SDS-PAGE (on 10\% gels), transferred to nitrocellulose 
membranes (Amersham; GE Healthcare, Chicago, IL, USA). Following blocking in 5\% non-fatty milk at room temperature for $60 \mathrm{~min}$, membranes were incubated overnight at $4^{\circ} \mathrm{C}$ with primary antibodies directed against $\beta$-actin (1:500; BM5422, Wuhan Boster Biological Technology, Ltd., Wuhan, China), B-cell lymphoma 2 (Bcl-2; 1:1,000; ab692), Bcl-2 associated X protein (Bax; 1:1,000; ab32503; both Abcam), Bcl-2 homologous antagonist/killer (Bak; 1:1,000; 12105, CST Biological Reagents Co., Ltd., Shanghai, China), Bcl-2-associated agonist of cell death (Bad; 1:1,000; 9268 , CST Biological Reagents Co., Ltd.), pro-caspase 3 and poly (ADP-ribose) polymerase (PARP; 1:1,000; 9532, CST Biological Reagents Co., Ltd.), respectively. The membranes were then washed three times with phosphate buffered saline with $0.05 \%$ Tween-20 buffer and incubated with horseradish peroxidase-conjugated secondary antibodies (1:5,000; 14709, CST Biological Reagents Co., Ltd.) for $1 \mathrm{~h}$ at room temperature. The blots were detected using chemiluminescence (Pierce; Thermo Fisher Scientific, Inc.) and an Odyssey Imaging System (Li-Cor Biosciences, Lincoln, NE, USA).

Glucose uptake assay. Basal glucose uptake was measured as previously reported for human cells in vitro (14). Briefly, HT-29 and SW620 cells were plated at $1 \times 10^{4}$ cells/well in 96-well cell culture plates and treated with $50 \mu \mathrm{g} / \mathrm{ml}$ AST at $37^{\circ} \mathrm{C}$ for $0,12,24$ and $48 \mathrm{~h}$. At every point in time, cells were treated with $100 \mu$ l of glucose-free DMEM culture medium containing $100 \mu \mathrm{g} / \mathrm{ml} \mathrm{2-NBDG}$ for $20 \mathrm{~min}$. The medium was subsequently replaced with $200 \mu$ l Hank's Balanced Salt Solution and the plate was centrifuged for $5 \mathrm{~min}$ at $30 \mathrm{x} \mathrm{g}$ (at room temperature). The fluorescence intensity was measured at $530 \mathrm{~nm}$ using a plate reader from Tecan Group Ltd. (Männedorf, Switzerland).

Glycolytic enzymes activity and lactate assay. $\mathrm{HK}$ and LDH activities were measured according to the manufacturer's instructions (Nanjing Jiancheng Bioengineering Institute (HK cat. no. A077-1 and LDH cat. no. A020-2; Nanjing, China). Cell lactate production was determined as previously reported in human cells (15). In brief, HT-29 and SW620 cells were collected, lysed via centrifugation at $6,000 \mathrm{x}$ g for $10 \mathrm{~min}\left(\right.$ at $\left.4^{\circ} \mathrm{C}\right)$. The supernatant was collected and total protein was examined using a BCA kit (Biyuntian, Shanghai, China).

Animals and treatment. A total of 32 male 9-week-old C57BL/6J mice (mean body weight of $21 \mathrm{~g}$; range, 18-25 g) were purchased from Shanghai SLAC Laboratory Animal Co., Ltd (Shanghai, China) and housed in individually ventilated cages (IVC; Tianhuan, Shanghai, China) supplied with filtered air and access to food and water. The mice were maintained under the following conditions: Temperature, $20-22^{\circ} \mathrm{C}$; relative humidity, 50-60\%; and a 12 -h light/dark cycle. All mice were acclimatized for 1 week prior to starting dextran sulfate sodium (DSS; molecular weight, 36-50 kDa; MP Biomedicals, Solon, OH, USA) administration. Mice were randomly divided into the following three groups: Mice were exposed to $2 \%$ DSS ( $\mathrm{n}=8$, positive control), $0.1 \mathrm{mg} / \mathrm{g}$ AST ( $\mathrm{n}=8$, negative control) and $0.1 \mathrm{mg} / \mathrm{g}$ AST treatment combined with $2 \%$ DSS in drinking water $(\mathrm{n}=8)$, respectively. The body weight of all mice was monitored daily. All mice were sacrificed 8 days following the administration of DSS. Colitis was induced by administration of $2 \%$ DSS dissolved in distilled water for 8 days. Once the colon length was measured, the distal colon was extracted and fixed in $4 \%$ paraformaldehyde for $24 \mathrm{~h}$ at room temperature. Paraffin-embedded colon tissues ( $5 \mu \mathrm{m}$ thick) were prepared for hematoxylin and eosin staining. The resulting tissue sections were stained with hematoxylin for $5 \mathrm{~min}$ and eosin for $3 \mathrm{~min}$ at room temperature. The sections were observed using a confocal microscope (LSM 5 PASCAL; Carl Zeiss AG, Oberkochen, Germany). The remaining colon tissues were embedded in paraffin or subjected to RNA and protein extraction. The procedures employed for the handing and care of animals were approved by the Renji Hospital Ethics Committee, Shanghai Jiao Tong University School of Medicine (Shanghai, China), and conformed to the national guidelines for animal use in research.

Statistical analysis. All analyses were performed using SPSS 17.0 statistical package (SPSS, Inc., Chicago, IL, USA). Data were expression as the mean \pm standard error of the mean. Data were compared using one-way analysis of variance followed by an LSD post-hoc test. $\mathrm{P}<0.05$ was considered to indicate a statistically significant difference.

\section{Results}

AST inhibits growth and proliferation in CRC cells. The MTT assay was performed to determine the growth inhibitory effect of AST on CRC cells. It was revealed that AST could inhibit the growth of CRC cells in a dose- and time-dependent manner (Fig. 1A and B). The $\mathrm{IC}_{50}$ values at $24 \mathrm{~h}$ in HT-29 and SW620 cells were $\sim 35$ and $46 \mu \mathrm{g} / \mathrm{ml}$, respectively. Thus, a final concentration of $50 \mu \mathrm{g} / \mathrm{ml}$ AST was used in all subsequent experiments unless otherwise specified. Following this, the inhibitory impact of AST on HT-29 and SW620 cell proliferation over an extended time interval was determined. To assess colony formation, cells were seeded in 6-well plates and treated with $50 \mu \mathrm{g} / \mathrm{ml}$ AST (cells treated with $25 \mu \mathrm{g} / \mathrm{ml}$ 5-Fu served as a positive control) for 2 weeks. Statistical analysis indicated that AST significantly reduced the number of colonies formed by CRC cells compared with the vehicle group (Fig. 1C and D). The results illustrated that AST has a directly inhibitory effect on CRC cell growth and viability over short- and long-term time points.

AST directly promotes apoptosis and the expression of apoptosis-associated genes in vitro. To determine whether the cytotoxic effects of AST are associated with the induction of apoptosis, flow cytometry was performed when AST was applied to cells. AST treatment significantly induced apoptosis in CRC cells (Fig. 2A and B). Furthermore, $\sim 34 \%$ of HT-29 cells and $\sim 31 \%$ of SW620 cells were apoptotic in response to AST. Notably, the expression levels of apoptosis-associated proteins confirmed the induction of apoptosis by AST, including the upregulation of Bax, Bad and $\mathrm{Bak}$, and the downregulation of Bcl-2, pro-caspase3, PARP (Fig. 2C). 

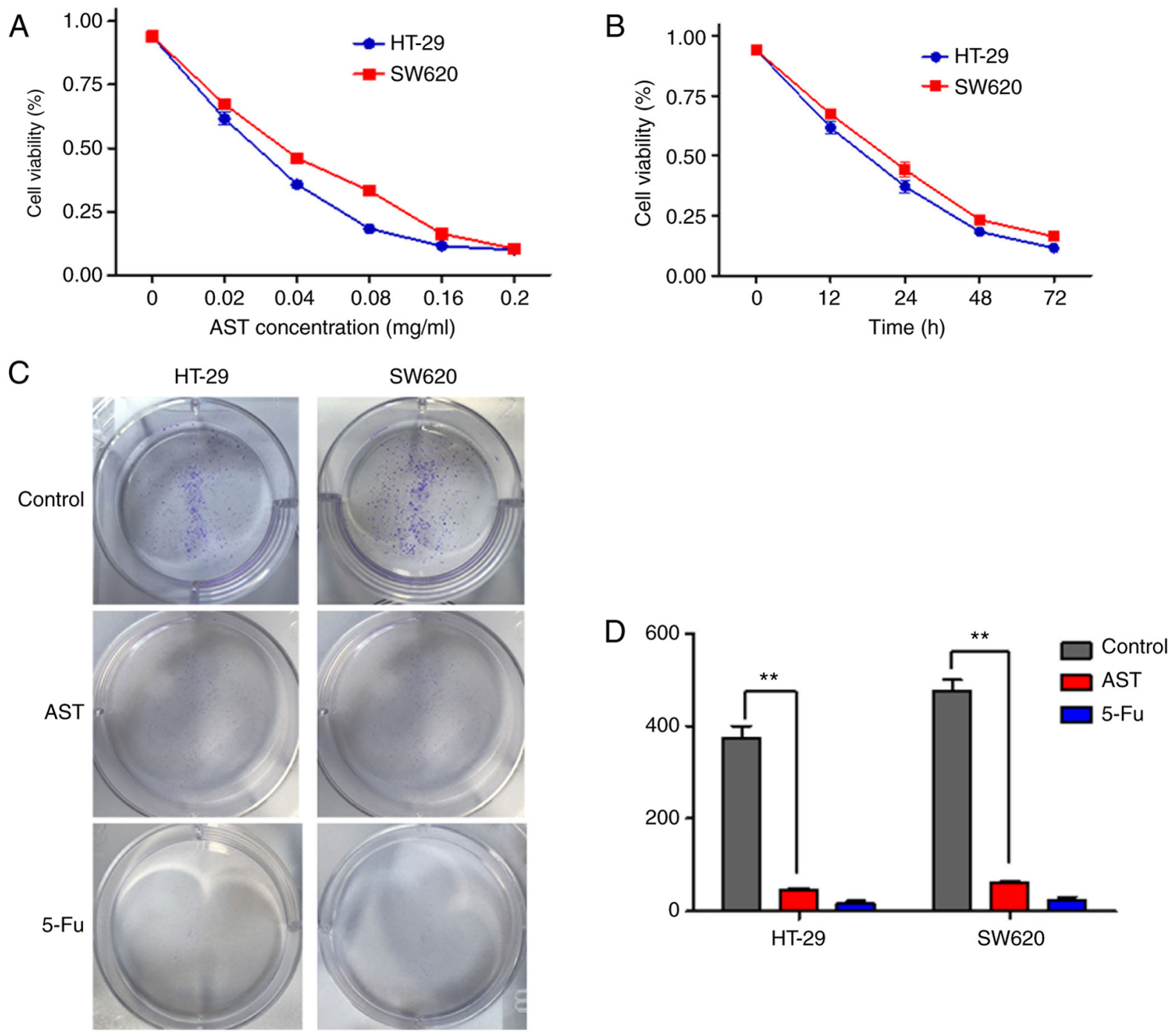

Figure 1. AST inhibits growth and proliferation in CRC cells. (A) HT-29 and SW620 cells were treated with increasing concentrations (0-0.2 mg/ml) of AST for $24 \mathrm{~h}$. Growth inhibition was assessed using the MTT assay. Control cells received McCoy's 5A or L-15 treatment and $0 \mathrm{mg} / \mathrm{ml}$ AST. (B) HT-29 and SW620 cells were treated with $50 \mu \mathrm{g} / \mathrm{ml}$ AST for different time intervals $(0,12,24,48$ and $72 \mathrm{~h})$ to assess cell proliferation. (C) Representative images of CRC cell colony formation. (D) Statistical analysis of the colony numbers. ${ }^{* *} \mathrm{P}<0.01$ as indicated. CRC, colorectal cancer; AST, Astragalus saponins; 5-Fu, 5-fluorouracil.

Glucose uptake and lactate production are decreased by AST treatment in CRC cells. To explore the impact of AST on glycolysis metabolism in CRC cells, glucose uptake and lactate levels were measured. As indicated in Fig. 3A, the glucose uptake of CRC cells decreased with AST treatment. Furthermore, lactate production was also reduced (Fig. 3B). In addition, the enzymes activities of HK2 and LDH-A in CRC cells were assessed. AST significantly reduced the enzymes activities of HK2 and LDH-A (Fig. 3C and D). The results illustrated that AST could inhibit aerobic glycolysis and the activities of glycolytic enzymes.

AST inhibits the expression levels of glycolytic enzymes in CRC cells. To verify whether the expression levels of glycolytic enzymes were also decreased by AST, western blot analysis and RT-qPCR were performed. The relative mRNA expression levels of LDH-A, Glut-1 and HK2 were significantly reduced by AST in SW620 cells (Fig. 4A, B and C). Interestingly, the expression of c-Myc, a primary oncogene of carcinogenesis that is overexpressed in various cancer types $(16,17)$, was significantly decreased in CRC cells treated with AST (Fig. 4D). In addition, western blot analysis revealed similar trends to RT-qPCR data (Fig. 4E).

AST attenuates the inflammatory response and tumor-like aerobic glycolysis in colitis mice model. To uncover the possible impact of AST on inflammation and glucose metabolism in vivo, a DSS-induced colitis mouse model was established. DSS is a well-known agent for inducing inflammation in order to identify the impact of inflammation on the pathogenesis and the clinical course of inflammation (18-20). In the DSS-induced colitis mouse model of the present study, compared with the combined treatment of AST and DSS, mice administered DSS alone were used as a positive control, and those with AST treatment alone served as a negative control. Body weight loss was observed over 8 days post-administration of DSS. In addition, colon length and histological analysis were observed 8 days post-administration of DSS. Following DSS treatment, the body weight and colon length of mice treated with DSS only were significantly reduced. Notably, there was only a slight decrease 

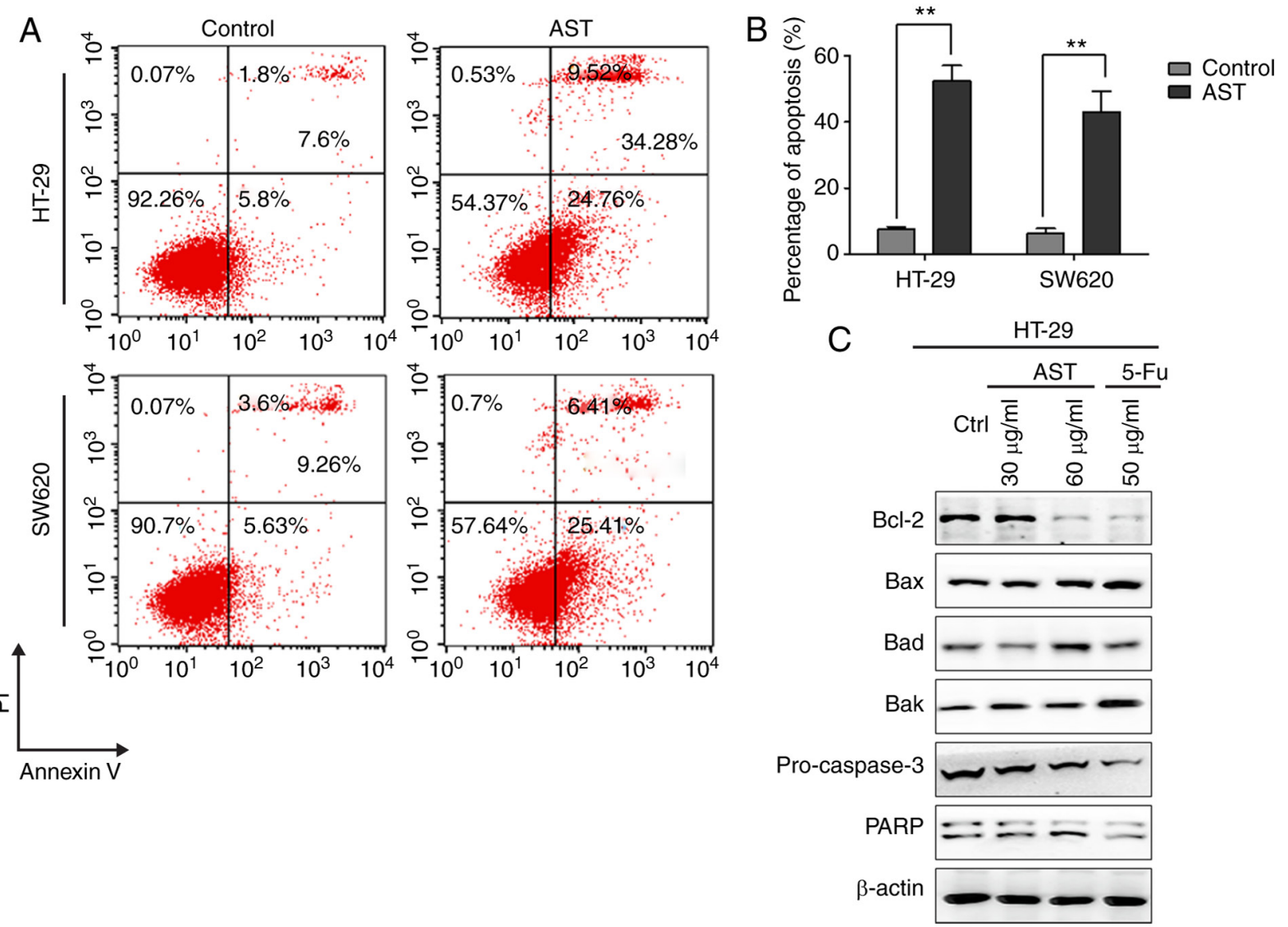

Figure 2. Induction of CRC cell apoptosis by AST treatment. (A) Representative images of CRC cell apoptosis. HT-29 and SW620 cells were treated with $50 \mu \mathrm{g} / \mathrm{ml}$ AST for $48 \mathrm{~h}$ and evaluated for apoptosis using Annexin V/propidium iodide double staining. Untreated cells were considered the control group. (B) Statistical analysis of the percentage of apoptotic CRC cells. ${ }^{* *} \mathrm{P}<0.01$ as indicated. (C) Western blot analysis of apoptosis-associated proteins in AST-treated HT-29 cells. CRC, colorectal cancer; AST, Astragalus saponins; 5-Fu, 5-fluorouracil; Bcl-2, B-cell lymphoma-2, Bax, Bcl-2-associated x protein; Bad, Bcl-2-associated agonist of cell death; Bal, Bcl-2 homologous antagonist/killer; PARP, poly (ADP-ribose) polymerase.
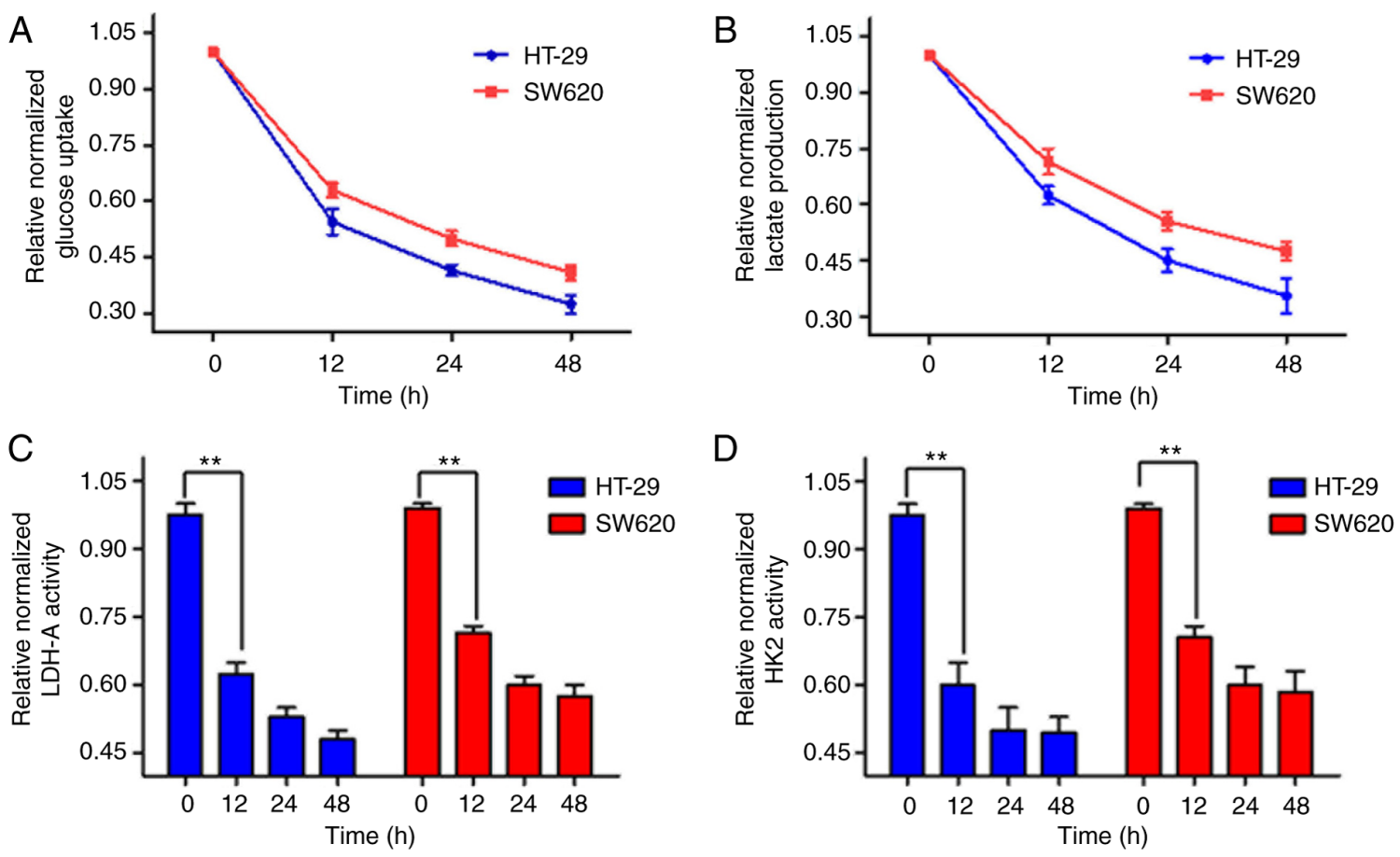

Figure 3. Alteration of glycolytic indexes in AST-treated CRC cells. HT-29 and SW620 cells were pre-treated with $50 \mu \mathrm{g} / \mathrm{ml}$ AST for different time intervals $(0,12,24$ and $48 \mathrm{~h}$ ). (A) Basal glucose uptake and (B) relative lactate production were measured at each time point. (C and D) Glycolytic enzyme activity analysis of LDH-A and HK2 in HT-29 and SW620 cells, respectively. The control groups were relative normalized value corresponding of $0 \mathrm{~h}$ in time gradient. ${ }^{* *} \mathrm{P}<0.01$ as indicated. CRC, colorectal cancer; AST, Astragalus saponins; LDH-A, lactate dehydrogenase A; HK2, hexokinase 2. 

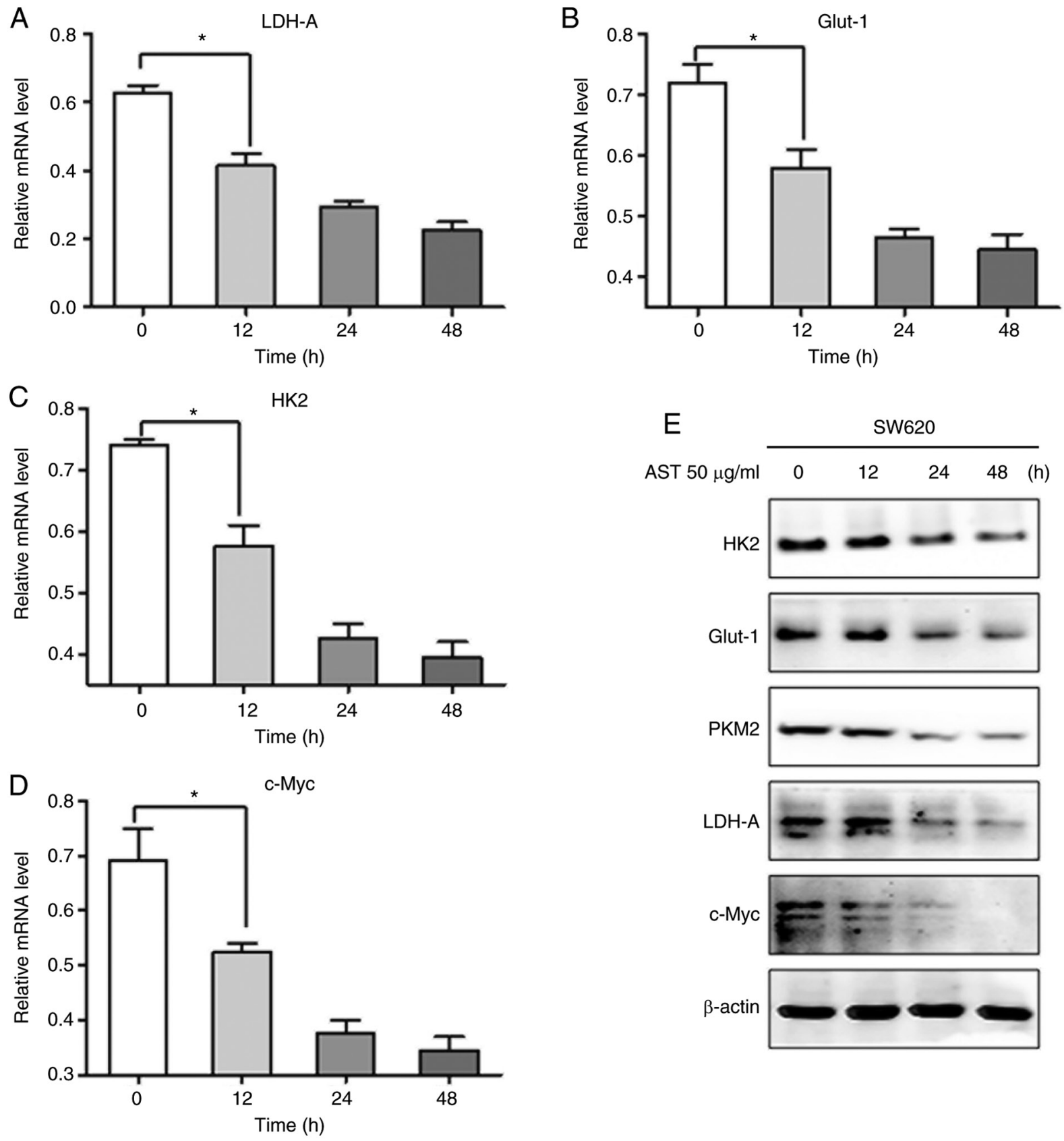

Figure 4. Downregulation of the expression of glycolytic enzymes by AST. HT-29 and SW620 cells were treated with $50 \mu \mathrm{g} / \mathrm{ml} \mathrm{AST} \mathrm{for} \mathrm{different} \mathrm{time} \mathrm{intervals}$ $\left(0,12,24\right.$ and $48 \mathrm{~h}$ ). mRNA expression levels of (A) LDH-A, (B) Glut-1, (C) HK2 and (D) c-Myc were decreased in SW620 cells treated with AST. ${ }^{*}<0.05$ as indicated. (E) Western blot analysis of key glycolytic enzymes in AST-treated SW620 cells. AST, Astragalus saponins; LDH-A, lactate dehydrogenase A; HK2, hexokinase 2; Glut-1, glucose transporter-1; PKM2, pyruvate kinase M2.

in the combined treatment group compared with control group and AST groups (Fig. 5A and B). In addition, the inflammatory response of the colonic mucosa was assessed using hematoxylin and eosin staining. As indicated in Fig. 5C, a large number of lobular necro-inflammatory cells were observed in the DSS group; however, this was reduced by AST treatment. The result suggests that AST treatment could attenuate the inflammatory response induced by DSS. Considering the decrease of body weight and the acute inflammatory reactions associated with DSS, it was proposed that glucose metabolism may be altered following inflammation enhancement. mRNA expression levels of c-Myc and glycolytic enzymes were increased in colon epithelial cells following 8-day DSS administration. However,
AST treatment significantly inhibited the tumor-like aerobic glycolysis (the high level of glycose uptake and lactate production) in mice with DSS-induced colitis (Fig. 5D), revealing almost the same trends that were indicated in the in vitro cell experiments.

\section{Discussion}

Astragalus is considered to be useful in traditional Chinese medicine and has been used as a treatment for almost all diseases believed to be caused by chi deficiency $(21,22)$. Previous studies have suggested that AST, a traditional Chinese medicine, has anti-tumor and apoptosis-inducing 

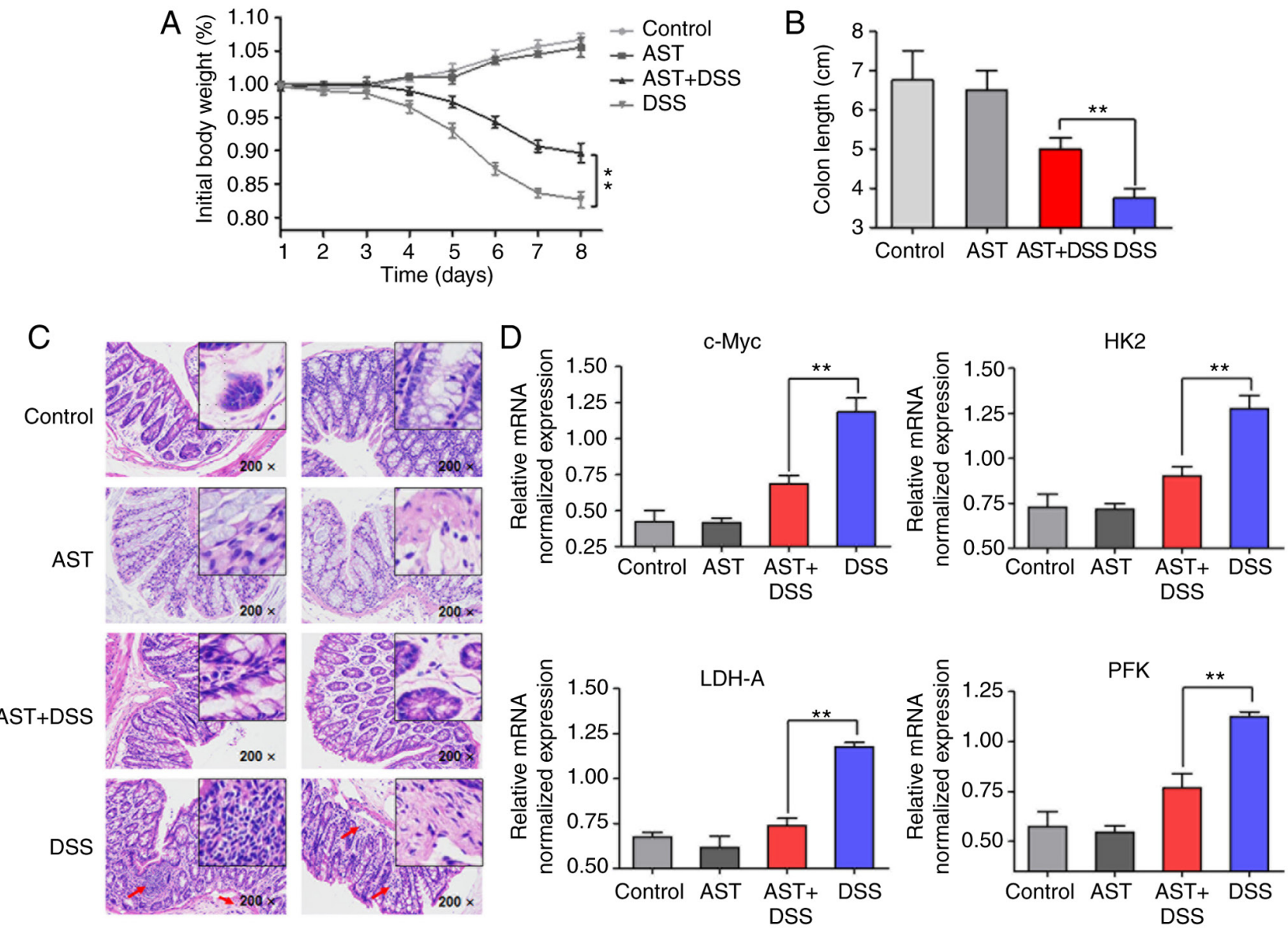

Figure 5. AST treatment attenuates the inflammatory response and tumor-like aerobic glycolysis in vivo. (A) Body weight change percentage was calculated in control, AST (negative control), DSS (positive control), and AST combined with DSS groups. (B) Colonic length changes of mice were presented. (C) Representative hematoxylin and eosin-stained colon sections. Magnifications of x 200 or x400 (zoomed in sections) were used. Lobular necro-inflammatory cells were labelled with red arrows. (D) c-Myc and glycolytic enzyme mRNA expression levels were decreased in AST-treated mice. Values were presented as the mean \pm standard error of the mean $(\mathrm{n}=8) .{ }^{* *} \mathrm{P}<0.01$ as indicated.

effects on various types of human cancer in vitro and in tumor xenografts (23-25). However, few studies have reported the impact of AST on metabolic alterations in CRC cells. To address this issue, the present study investigated a series of glycolysis metabolism and growth condition alterations in CRC cells treated with AST.

In the present study, the cytotoxic activity of AST was determined in CRC cells. The results illustrated that AST could inhibit growth and proliferation of CRC cells, and that AST could directly induce apoptosis in vitro. Western blot analysis of apoptosis-associated proteins further confirmed the induction of apoptosis by AST. For instance, AST decreased the expression of anti-apoptotic protein Bcl-2, PARP and pro-caspase 3, and increased the expression of the pro-apoptotic proteins Bax, Bak and Bad in CRC cells. In the 1920 s, Warburg hypothesized that there was a trend toward an increased rate of glycolysis in a variety of cancer cells, triggering the excessive production of lactic acid from glucose, which was termed the 'Warburg effect' $(26,27)$. Accordingly, the impact of AST on glycolysis metabolism was investigated in the present study. A series of indexes of aerobic glycolysis, including glucose uptake, lactate production and the activity of glycolytic enzymes, were all decreased. Western blot analysis and RT-qPCR data revealed similar trends. In addition, the present study established a DSS-induced colitis mouse model, with the objective of exploring the possible impact of AST on the inflammatory response and glucose metabolism. The results suggested that AST could effectively attenuate the inflammatory response and inhibit tumor-like aerobic glycolysis, indicating that AST may have the capacity to resist inflammation and maintain normal glucose homeostasis.

Furthermore, evidence has suggested the multifaceted oncogene, c-Myc, exerts important regulatory impacts on multiple aspects of cell transformation $(16,28,29)$. It was reported that c-Myc could activate the expression of LDH-A and increase lactate production (30). The present study indicated that AST decreased c-Myc expression in CRC cells. The results suggested that AST-induced inhibition of growth and aerobic glycolysis of CRC cells may be associated with the suppression of c-Myc; specifically, AST may inhibit the Warburg effect through c-Myc suppression.

In conclusion, the present findings indicated that AST may be able to inhibit CRC development and attenuate aerobic glycolysis, which may be associated with c-Myc downregulation. The present study may provide a new therapeutic strategy for clinical treatment of CRC.

\section{Acknowledgements}

Not applicable. 


\section{Funding}

The present study was supported by a grant from the Shanghai Medical Key Specialist Construction plans (grant no. ZK2015B10).

\section{Availability of data and materials}

The datasets used and/or analyzed during the current study are available from the corresponding author on reasonable request.

\section{Authors' contributions}

ZS conceived and designed the study. HG, BW and JW performed the drugs preparation and treatments, western blot analysis and mice model, and were major contributors in writing the manuscript. JZ and WY performed the reverse transcription quantitative polymerase chain reaction and analyzed the data. All authors read and approved the final manuscript.

\section{Ethics approval and consent to participate}

The present study was approved by Renji Hospital Ethics Committee, Shanghai Jiaotong University School of Medicine (Shanghai, China).

\section{Patient consent for publication}

Not applicable.

\section{Competing interests}

The authors declare that they have no competing interests.

\section{References}

1. Tenesa A and Dunlop MG: New insights into the aetiology of colorectal cancer from genome-wide association studies. Nat Rev Genet 10: 353-358, 2009.

2. Siegel RL, Miller KD, Fedewa SA, Ahnen DJ, Meester RGS, Barzi A and Jemal A: Colorectal cancer statistics, 2017. CA Cancer J Clin 67: 177-193, 2017.

3. Potack J and Itzkowitz SH: Colorectal cancer in inflammatory bowel disease. Gut Liver 2: 61-73, 2008.

4. Ullman TA and Itzkowitz SH: Intestinal inflammation and cancer. Gastroenterology 140: 1807-1816, 2011.

5. Wei H, Sun R, Xiao W, Feng J, Zhen C, Xu X and Tian Z: Traditional Chinese medicine Astragalus reverses predominance of Th2 cytokines and their up-stream transcript factors in lung cancer patients. Oncol Rep 10: 1507-1512, 2003.

6. McCulloch M, See C, Shu XJ, Broffman M, Kramer A, Fan WY, Gao J, Lieb W, Shieh K and Colford JM Jr: Astragalus-based Chinese herbs and platinum-based chemotherapy for advanced non-small-cell lung cancer: Meta-analysis of randomized trials. J Clin Oncol 24: 419-430, 2006.

7. Wu CY, Ke Y, Zeng YF, Zhang YW and Yu HJ: Anticancer activity of Astragalus polysaccharide in human non-small cell lung cancer cells. Cancer Cell Int 17: 115, 2017.

8. Ye MN and Chen HF: Effects of Astragalus injection on proliferation of basal-like breast cancer cell line MDA-MB-468 Zhong Xi Yi Jie He Xue Bao 6: 399-404, 2008 (In Chinese).
9. Deng Y and Chen HF: Effects of Astragalus injection and its ingredients on proliferation and Akt phosphorylation of breast cancer cell lines. Zhong Xi Yi Jie He Xue Bao 7: 1174-1180, 2009 (In Chinese).

10. Wang T, Xuan X, Li M, Gao P, Zheng Y, Zang W and Zhao G: Astragalus saponins affect proliferation, invasion and apoptosis of gastric cancer BGC-823 cells. Diagn Pathol 8: 179, 2013.

11. Cassileth BR, Rizvi N, Deng G, Yeung KS, Vickers A, Guillen S, Woo D, Coleton M and Kris MG: Safety and pharmacokinetic trial of docetaxel plus an Astragalus-based herbal formula for non-small cell lung cancer patients. Cancer Chemother Pharmacol 65: 67-71, 2009.

12. Dugoua JJ, Wu P, Seely D, Eyawo O and Mills E: Astragalus-containing Chinese herbal combinations for advanced non-small-cell lung cancer: A meta-analysis of 65 clinical trials enrolling 4751 patients. Lung Cancer (Auckl) 1: 85-100, 2010.

13. Ma XQ, Shi Q, Duan JA, Dong TT and Tsim KW: Chemical analysis of Radix Astragali (Huangqi) in China: A comparison with its adulterants and seasonal variations. J Agric Food Chem 50: 4861-4866, 2002.

14. Schlaepfer IR, Hitz CA, Gijón MA, Bergman BC, Eckel RH and Jacobsen BM: Progestin modulates the lipid profile and sensitivity of breast cancer cells to docetaxel. Mol Cell Endocrinol 363: 111-121, 2012.

15. Zhan P, Wang Y, Zhao S, Liu C, Wang Y, Wen M, Mao JH, Wei G and Zhang P: FBXW7 negatively regulates ENO1 expression and function in colorectal cancer. Lab Invest 95: 995-1004, 2015.

16. Stine ZE, Walton ZE, Altman BJ, Hsieh AL and Dang CV: MYC, metabolism, and cancer. Cancer Discov 5: 1024-1039, 2015.

17. Miller DM, Thomas SD, Islam A, Muench D and Sedoris K: c-Myc and cancer metabolism. Clin Cancer Res 18: 5546-5553, 2012.

18. Parang B, Barrett CW and Williams CS: AOM/DSS model of colitis-associated cancer. Methods Mol Biol 1422: 297-307, 2016.

19. Antoniou E, Margonis GA, Angelou A, Pikouli A, Argiri P, Karavokyros I, Papalois A and Pikoulis E: The TNBS-induced colitis animal model: An overview. Ann Med Surg (Lond) 11: 9-15, 2016.

20. Qu D, Shen L, Liu S, Li H, Ma Y, Zhang R, Wu K, Yao L, Li J and Zhang J: Chronic inflammation confers to the metabolic reprogramming associated with tumorigenesis of colorectal cancer. Cancer Biol Ther 18: 237-244, 2017.

21. Wang XM, Yu RC and Wang YT: Study on advanced non-small cell lung cancer patients with Qi deficiency and blood stasis syndrome. Zhongguo Zhong Xi Yi Jie He Za Zhi 14: 724-726, 1994 (In Chinese).

22. Yuan F, Zhou Y, Jiang Y, Liu R, Li JZ, Xie YK, Li XM and Dai F: Therapeutic effect and apoptosis mechanism of lung-tonifying and expectorant decoction on lung cancer rats with Qi deficiency and blood stasis. Asian Pac J Trop Med 8: 983-988, 2015.

23. Dong JC, Dong XH and Zhao FD: Therapeutic effects of Astragalus injection on lewis lung cancer in mice. Zhonghua Zhong Liu Za Zhi 28: 272-273, 2006 (In Chinese).

24. Zou YH and Liu XM: Effect of astragalus injection combined with chemotherapy on quality of life in patients with advanced non-small cell lung cancer. Zhongguo Zhong Xi Yi Jie He Za Zhi 23: 733-735, 2003 (In Chinese).

25. Na D, Liu FN, Miao ZF, Du ZM and Xu HM: Astragalus extract inhibits destruction of gastric cancer cells to mesothelial cells by anti-apoptosis. World J Gastroenterol 15: 570-577, 2009.

26. Warburg O: On the origin of cancer cells. Science 123: 309-314, 1956.

27. Warburg O: On respiratory impairment in cancer cells. Science 124: 269-270, 1956.

28. Dang CV: MYC on the path to cancer. Cell 149: 22-35, 2012.

29. Hsieh AL, Walton ZE, Altman BJ, Stine ZE and Dang CV: MYC and metabolism on the path to cancer. Semin Cell Dev Biol 43: $11-21,2015$

30. Shim H, Dolde C, Lewis BC, Wu CS, Dang G, Jungmann RA, Dalla-Favera R and Dang CV: c-Myc transactivation of LDH-A: Implications for tumor metabolism and growth. Proc Natl Acad Sci USA 94: 6658-6663, 1997. 